\section{Hints On Costing Lab Services}

Timothy Morken

Centers for Disease Control, Atlanta, GA

[Ed. Note: While the following is from the author's experience in a histology lab, the information will be helpful for any type of microscopy service lab, with suitable changes in the details.

Costing laboratory services is a huge job and takes a long time to do properly. I've done this many times, but only once from scratch and that one time took six months to finalize. The whole process depends on what the administration really wants and what you have to work with. There should be guidelines given and help forthcoming from the administration, as the results for the lab won't mean much if everyone does it a different way! I wouldn't even start figuring things until the administration gives guidelines and holds some meetings on how it is to be done.

For instance, do they want the entire cost of materials, including shipping and ordering time costs, included? Will they provide full costs of employees, including benefits? Labor costs will be far more than all other costs put together! Are costs of procedures to be based on time taken only for each procedure, or on all the time actually worked? Surprisingly, about one third to one-half of the time in the lab is "non-productive" - that is, the techs aren't working on a specific task. Are fixed costs (building, electricity, refrigerators, etc.) and support costs (cleaning crew, secretaries) included? If equipment is included, depreciation and replacement costs for the equipment need to be figured in.

To arrive at the most accurate figure, the amounts of reagents used for each test, contributory costs of equipment (what percentage of the cryostat cost goes to rush frozen sections and how much to immunofrozen sections?), and supplies (glassware, gloves, blades) will have to be calculated. This requires access to accurate figures of items ordered from the central warehouse. Are those figures available?

Must any "professional" time (e.g., pathologists time) be included?

Must the cost of a central computer system be included?

How is the information to be used? Are a few basic charges that cover everything needed, or is it necessary or preferable to tack on extra charges for everything done?

This all assumes the administration wants the true cost which, frankly, some administrators would rather not know!

This is what I do:

1) Figure labor costs for techs and for pathologists for one year and come up with a per-minute labor charge (an average labor cost perminute), with separate costs for technicians and pathologists. The per-minute cost is used because labor costs in all procedures will be figured in "minute" terms.

NOTE: the pathologists' cost I have used has been applied to things like looking at slides, not making a diagnosis. Some costs like this are figured in other charge codes, so look into that. It is possible that no pathologists' (or equivalent) costs will be put into the mix.

2) Make a cost sheet for each procedure. It is best to decide on a basic level of procedure and then add to it for specialized tests. For

\title{
PHOENIX
}

\section{Unleash the Power of X-ray Microanalysis}

Setting the standard in $x$-ray microanalysis, Phoenix, the most powerful system available offers the next generation of EDS...today. Phoenix delivers the ultimate in performance capabilities plus

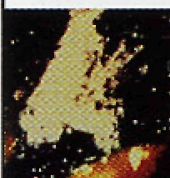
reliability, upgradability and flexibility. This user-friendly, easy-to-operate work station with 32-bit software is available for Windows ${ }^{\circledR}$ NT or Windows '98. Accelerate your performance with Phoenix...the most powerful tool in microanalysis!

- Power - true standardless, high quality quantification regardless of the matrix, plus the ability to achieve a high speed quantitative map in the same time as a gross map, along with a digital pulse processor.

- Simplicity - full quantitative results in seconds at the click of a mouse. Generate reports easily and import into other programs.

- Support - remote application and service support through REMAC.

To power-up your microanalysis capabilities, call EDAX today.

91 McKee Drive

Mahwah, NJ 07430-9978

Tel. Office: $201-529-4880$

Fax: 201-529-3156
Ringbaan Noord 103, P.0. Box 4144

5004 JC Tilburg, The Netherlands

Tel. Office: $+31-13-5364000$

Fax: $+31-13-5356279$
13-37, Kohnan 2-chome, Minato-ku Tokyo 108, Japan

Tel. Office; 81-3-3740-5172

Fax: $81-3-3740-5190$

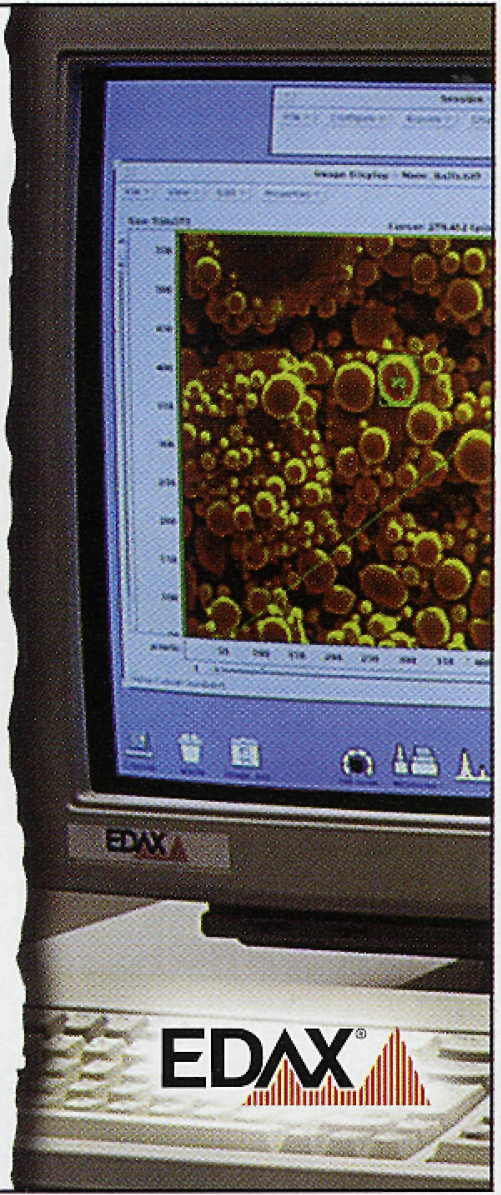


instance, I base everything on a single Hematoxylin \& Eosin (H\&E) produced from one block. That is the basic cost, since an H\&E is done on almost everything. All the accessioning, grossing, processing, embedding and sectioning costs are rolled into that one H\&E from one block Extra H\&E's are extra cost. Special stains are an add-on, as are immunostains. Gross anatomy photos are an add-on. Frozen sections are an add-on, etc.

3) Figure supply (materials) costs. These may be best broken down into warehouse items and outside orders.

4) Figure fixed costs. Find and include all your equipment!

5) Figure add-on costs. Add up labor (time in minutes $X$ labor cost per minute), materials, reagents and fixed costs for an average procedure. Sometimes, as with frozen sections or other special procedures, it makes sense to figure a one-specimen charge and then another "extra specimen" charge or extra slide charge.

After completing all this, the cost of doing every combination of tests can be accurately added up. What results is a basic cost for producing a H\&E and separate "add-on" costs for every test one does. This is obviously for a histology lab. For other microscopy labs, substitute the standard procedure used for the H\&E (i.e., ion-milling a TEM sample in a materials lab, or the first grid from a block in a biology TEM lab). This means exact costs can be determined by picking and choosing the tests used, or standard charge sets can be made up.

It is unlikely that the administration will want to charge for every little thing done because it's too unwieldy, unless there is a great database in use and they can handle multiple charges. Instead, it would probably be best to come up with charge sets for the average test batteries. In this case, be very careful to account for a true average of tests in a battery (Silver stains cost a lot more than Periodic acid Schiff's stains, and some antibodies are extremely expensive). Also, how does the administration want to charge for special procedures, such as frozen sections? Especially since sometimes one is done, and sometimes fifteen are done on one patient (or sometimes one TEM grid and sometimes fifteen grids are done on one specimen).

\section{A few tips:}

The cost of dyes is negligible in figuring special stain costs: labor will be a thousand times more than the dye cost, so don't bother with figuring exactly how much is used. With silver stains, however, the reagent cost is very significant.

Remember to count wasted materials, such as slides cut but not used. They really add up (a slide costs about 10 cents, assuming cheap glass).

Labor is easier to figure if converted to a minute-based figure. That is, what is the average labor cost per minute?

In order to make it easier next time, set up a system to track all these costs so that they can be called up at any time in the future. Learn to use a database program for this.

Good luck!

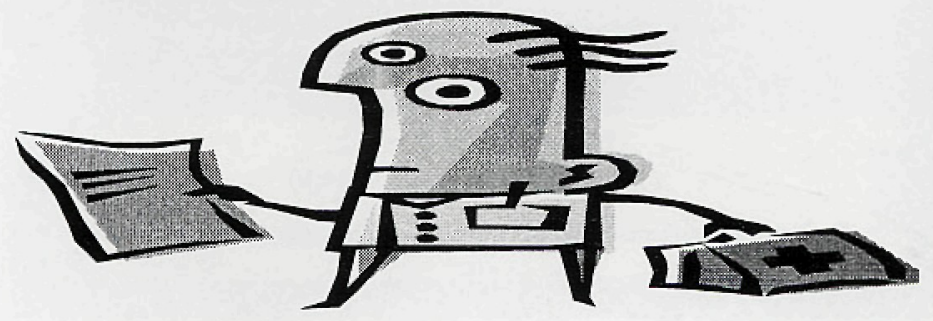

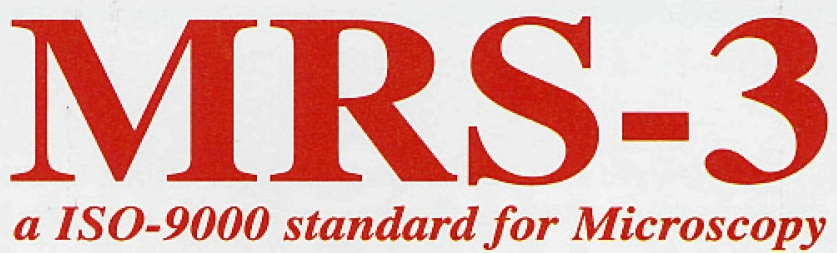

Our second generation, traceable, magnification reference standard for all types (SEM, Optical, STM, AFM, etc.) of microscopy. Usable from $10 \mathrm{X}$ to $50,000 \mathrm{X}$ with pitch patterns from $2 \mu \mathrm{m}$ $( \pm 0.1 \mu \mathrm{m})$ to $8 \mathrm{~mm}( \pm 0.25 \mu \mathrm{m})$. Pattern height traceable to $0.1 \mathrm{~mm}$ $\pm .001 \mu \mathrm{m}$. New patterns for parti-

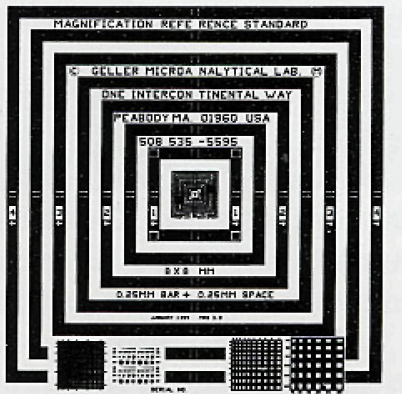
cle size counting (circles, squares and rectangles) and chemical imaging. Several hundred MRS's have been sold to date.

Send for our free RESOURCE GUIDE which discusses magnification measurement, error determination and calibration procedures.

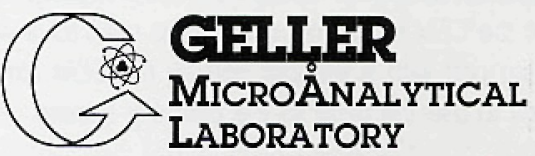

426 BOSTON STREET (RT. 1) * TOPSFTED, MA 01983 978/887-7000 *978/887-6671 * jg@gellermicro.com http://www.gellermicro.com

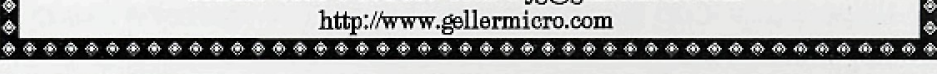

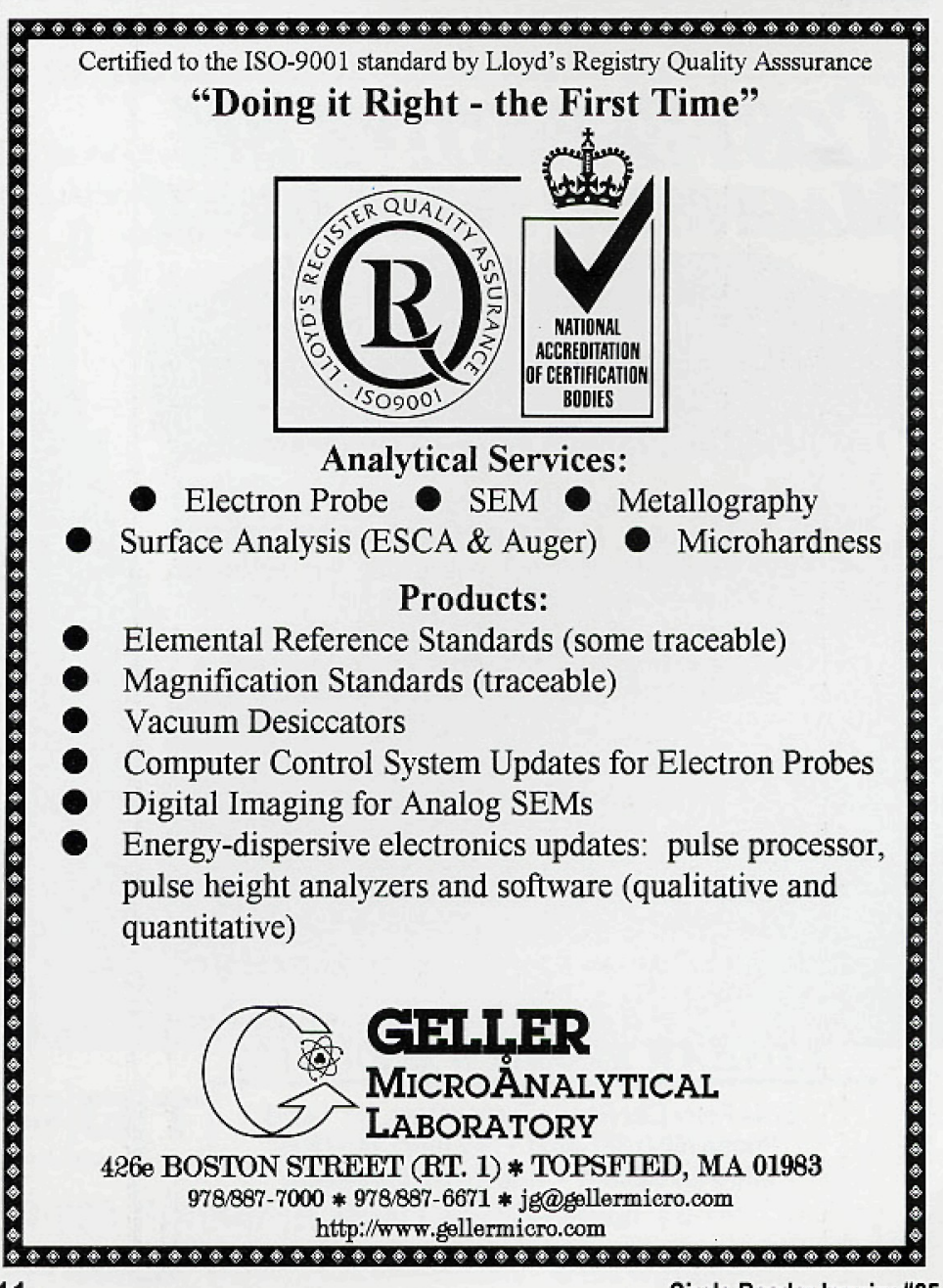

